\title{
Redesigning Skin Cancer Early Detection and Care Using a New Mobile Health Application: Protocol of the SKIN Research Project, a Randomised Controlled Trial
}

\author{
Monika Janda ${ }^{a}$ b Caitlin Horsham ${ }^{a}$ b Uyen Koh ${ }^{a}$ Nicole Gillespie \\ Lois J. Loescher $^{d}$ Dimitrios Vagenas $^{b}$ H. Peter Soyer ${ }^{e}, f$
}

\begin{abstract}
${ }^{a}$ Centre of Health Services Research, Faculty of Medicine, The University of Queensland, Brisbane, QLD, Australia;
${ }^{\mathrm{b}} \mathrm{S}$ chool of Public Health and Social Work, Institute for Health and Biomedical Innovation, Queensland University of Technology, Brisbane, QLD, Australia; ' $M e l$ and Enid Zuckerman College of Public Health, Colleges of Nursing and Public Health, The University of Arizona, Tucson, AZ, USA; ' $U Q$ Business School, The University of Queensland, Brisbane, QLD, Australia; ${ }^{e}$ Dermatology Research Centre, The University of Queensland, The University of Queensland Diamantina Institute, Translational Research Institute, Brisbane, QLD, Australia; ${ }^{\mathrm{f}}$ Dermatology Department, Princess Alexandra Hospital, Brisbane, QLD, Australia
\end{abstract}

\section{Keywords}

Melanoma · Skin cancer · Mobile teledermoscopy ·

Melanoma apps

\begin{abstract}
Patients often detect melanoma themselves; therefore, regular skin self-examinations (SSEs) play an important role in the early detection and prompt treatment of melanoma. Mobile teledermoscopy is a technology that may facilitate consumer SSEs and rapid communication with a dermatologist. This paper describes the planned randomised controlled trial of an intervention to determine whether mobile technologies can help improve the precision of SSE in consumers. A randomised controlled trial will be conducted to evaluate mobile teledermoscopy-enhanced SSE versus naked-eye SSE. Participants in each group will conduct three home whole-body SSEs at baseline, 1 and 2 months, then present for a clinical skin examination (CSE) by a doctor after the 2-month SSE. Specifically, participants will identify skin le-
\end{abstract}

() 2018 S. Karger AG, Basel

E-Mail karger@karger.com

www.karger.com/drm sions that meet the AC (asymmetry and colour) rule for detecting a suspicious skin spot. The primary outcomes are sensitivity and specificity of the skin lesions selected by the participants as needing attention by a doctor, compared to the clinical diagnosis by the dermatologist that will serve as the reference standard for this analysis. For the mobile teledermoscopy-enhanced SSE group, researchers will assess the number, location and type of lesions (1) sent by the participant via mobile teledermoscopy, (2) found at CSE or (3) missed by the participant. For the naked-eye SSE group, researchers will assess the number, location and type of lesions (1) recorded on their body chart by the participant, (2) found at CSE or (3) missed by the participant. Secondary outcomes are based on participants' self-reported data via online questionnaires.

(c) 2018 S. Karger AG, Basel

Trial registration No. ACTRN12616000989448.

This article is part of the Nevi Article Series
Monika Janda

Centre for Health Services Research, Faculty of Medicine, The University of Queensland Building 33, Princess Alexandra Hospital Campus

Woolloongabba, QLD 4102 (Australia)

E-Mail m.janda@uq.edu.au 


\section{Introduction}

Melanoma is the third most common cancer in men and women in Australia, causing about 1,500 potentially avoidable deaths each year [1]. Patients with melanomas $<1 \mathrm{~mm}$ in thickness have a 5-year survival rate of 98.7\%; prognosis worsens with increasing thickness at diagnosis $[2,3]$, so early diagnosis is critical. Further improvements in early detection methods and technologies are necessary to stem the burden of melanoma on the Australian population in terms of morbidity, mortality and costs. In the absence of a population-based screening programme, melanoma early detection relies heavily on people being vigilant to skin changes and then rapidly presenting to a doctor once they notice a change $[4,5]$. Currently doctors in Australia excise about 20 benign lesions for each melanoma [6].

Even though the prevalence of people self-reporting at least 1 skin self-examination (SSE) within the previous 3 years has increased from $34 \%$ in 2004 [7] to 55\% in 2015 [8], many people do not conduct SSE at the recommended interval or thoroughly (i.e., they look only at easy-tosee areas of the body). Current SSE guidelines also do not differ depending on whether a person has few or many moles; thus, reports on the sensitivity of SSE vary widely, from 25 to $93 \%[9,10]$. Most studies contributing to the evidence for SSE are small, use a non-randomised prepost study design or test accuracy of detecting change in skin lesions which are artificially enlarged [9]. In the study by Muhn et al. [11], artificial change was examined by artificially increasing mole size on participants' backs, and then they were asked to detect any increases in size. Key studies on the role that technology could play in enhancing SSE show consumers recognise melanoma better from dermoscopic images than from clinical photographs [12]. Furthermore, given the different visual task that is needed for SSE depending on whether a person has few or many moles, it is surprising there is no previous study of consumers' use of a 2-level process to SSE, similar to that advocated for clinical skin examinations (CSEs) by a doctor [13-15]. Marghoob and Scope [14] recommended doctors to first consider the patient at a macrolevel (patient phenotype, skin cancer risk factors, personal and family history, and type and number of skin lesions on the whole body), then at a microlevel (given the patient's macrolevel risk, pattern analyses of individual lesions). These authors concluded that the 2-step approach can improve the robustness of recognising melanomas [14].

A 2-step approach thus seems sensible for SSE, with step 1 similar to the current naked-eye approach (macro- level) instructing consumers to consider their body overall, given the number and type of their skin lesions in addition to their personal and familial skin cancer history. Step 2 (microlevel) guides the mobile teledermoscopyenhanced SSE appropriate for their macrolevel risk either looking at each skin lesion (if few are present) or selecting the most unusual ones ("ugly duckling sign") if they have many skin lesions. Mobile teledermoscopy-enhanced SSE combines a dermoscope with a smartphone's camera and using an app guides the user through imaging moles. Currently there are apps available that allow people to take and send photos of moles; however, these are highly variable in sophistication and costs [16].

While the present study authors $[17,18]$ and others [19-21] have conducted successful feasibility studies of mobile teledermoscopy-enhanced SSE and have shown excellent diagnostic agreement between teledermatological and clinical diagnosis $(\kappa=0.90)[17-19,21]$, no study has compared directly whether adding mobile teledermoscopy to SSE can improve outcomes compared to naked-eye SSE, whether accuracy improves over time and how to best integrate mobile teledermoscopy-enhanced SSE into consumer instructions and materials, and clinical practice. Integration also includes assessing costs of the two approaches. We describe in this paper the protocol for a randomised controlled trial, called the SKIN (SKin INnovation) Research Project, testing whether or not mobile teledermoscopy can aid SSE outcomes in consumers.

\section{Methods and Analysis}

\section{Design}

The SKIN Research Project is a randomised controlled trial conducted in Brisbane, Australia. Potential persons who meet the eligibility criteria described below and consent to participate will be randomised into either a control or intervention group (ratio = 1:1). A random allocation sequence will be generated using Internet-based randomisation software integrated into the REDCap (Vanderbilt University) research database system. REDCap utilises allocation concealment keeping the research staff unaware of which group the participant is randomised into. Study data will be collected and managed using REDCap electronic data capture tools, which provide a full log of data entry.

This study has been approved by the Queensland University of Technology's (QUT) Human Research Ethics Committee on 20 June 2016 (QUT approval No.: 1400000807). The trial was registered prospectively with the Australian and New Zealand Clinical Trials Registry (Registration No.: ACTRN12616000989448).

Study Aims

The main aim of this study is to assess whether mobile teledermoscopy-enhanced SSE can help improve the precision of SSE and build an evidence base for its effectiveness.
Janda/Horsham/Koh/Gillespie/Loescher/ Vagenas/Soyer 
- Objective 1: compare number, type and location of spots or moles selected with naked-eye SSE (usual care) versus mobile teledermoscopy-enhanced SSE

- (a) Sensitivity and specificity of (i) naked-eye SSE and (ii) mobile teledermoscopy-enhanced SSE by consumers, compared with the assumed gold standard of clinical diagnosis by the dermatologist

- (b) Sensitivity and specificity of examinations i versus ii

Further analysis will involve conducting health economic analysis alongside the randomised trial and investigating business models for teledermoscopy and pathways on how to integrate it into practice.

\section{Study Participants}

Participants will be eligible for the study if they meet all of the following criteria:

- Are 18 years or older

- Have at least 2 risk factors for skin cancer (high-risk phenotype including light skin and hair, skin that never or rarely tans and always or mostly burns, a family history of melanoma or a personal history of skin cancer [excluding melanoma within the last 5 years], history of skin excisions or many naevi, and residing in Queensland which has a high UV exposure all year round)

- Own or have access to an iPhone 5, 6, 7 or 8 compatible with the dermoscope attachment

- Reside in close proximity or willing to travel to the QUT, Kelvin Grove or the University of Queensland, Woolloongabba, to attend a visit for a whole-body CSE by the study dermatologist

- Have a partner, relative or friend available to aid with home SSE, especially looking at hard-to-reach body areas including the back and back of the neck

Excluded are persons with a history of melanoma within the past 5 years as research suggests these patients may be under regular screening and surveillance by a doctor, which would likely influence their SSE behaviour.

\section{Recruitment Procedures}

Recruitment of participants will be directed to a study-specific website through traditional digital media. Potential participants can securely enter their contact details via a website contact form. They will then receive an eligibility questionnaire (criteria specified above) by e-mail that includes the participant information sheet and online consent form. Once the eligibility questionnaire is complete and eligibility is confirmed, the research team will phone and enrol participants. During this call, study staff will review study procedures, schedule the participant for their in-person whole-body CSE and obtain their postal address to send out the study packages.

\section{Intervention and Comparator}

Figure 1 illustrates the procedures of the trial. Participants will be randomised to either a mobile teledermoscopy-enhanced SSE group (intervention) or naked-eye SSE (control). Participants in both groups will receive detailed instructions on how to detect melanoma or other skin cancers and conduct a whole-body SSE. They will be asked to complete 3 whole-body SSEs in their home at baseline, 1 and 2 months later. During the SSE, participants in both groups are asked to detect skin lesions "they do not like the look of" that may be suspicious for skin cancer, to follow the AC (asymmetry and colour) rule and then note them on a body chart.
The body chart is either (i) completed electronically in Microsoft Word and e-mailed back to the research team or (ii) printed, completed on paper, then photographed and e-mailed back to the research team. Intervention group participants will also take a photo of their suspicious skin lesions via the study app and e-mail the image to the research team for telediagnosis by the dermatologist. After completion of the 3 home SSEs, participants will be asked to attend the study clinic within 3 months for an in-person CSE.

\section{Intervention Group (Mobile Teledermoscopy-Enhanced SSE)}

Participants will be mailed the dermoscope attachment (Handyscope FotoFinder Systems), web-based instructions on how to download the Handyscope App, instructions on what features to look for using the AC rule developed by Luttrell et al. [22] and directed to a step-by-step instructional video. The AC rule used for both nakedeye and dermoscope assessment is a simpler tool that laypersons may use to identify a melanoma. SSEs should be aided by another person (i.e., partner, a relative or close friend) to allow for operating the camera in hard-to-reach areas of the body, as specified in the eligibility criteria. Participants/partners will follow these procedures:

1 Conduct a whole-body, naked-eye SSE

2 Use the Handyscope App to evaluate suspicious-looking skin lesions using the $\mathrm{AC}$ rule

3 Use the Handyscope App to photograph skin lesions at baseline, 1 and 2 months

4 E-mail the images to the study team through the Handyscope App

5 Complete an online body chart to pinpoint the location of lesions identified during SSE for use during the CSE. The body chart includes numbering skin lesions they think need management by a doctor and indicating urgency (index of suspicion) for management (from $1=$ not at all concerned to $10=$ very concerned). The body chart also includes other notes, such as history of each lesion, whether it changed or symptoms, i.e. itch, pain, bleeding)

Participants receive e-mail, text or phone reminders if their skin checks are overdue at 1 and 2 months.

\section{Telediagnosis}

For the intervention group, a telediagnosis will be completed after every home SSE by the study dermatologist (H.P.S.). Along with the telediagnosis, the dermatologist rates the likelihood of malignancy (from $1=$ very unlikely to $5=$ very likely), recommendation to excise (from $1=$ not at all to $5=$ very strongly) and management strategy (none, observation, treatment (surgical or nonsurgical), referral). If any lesions are suspected to be a melanoma or keratinocyte skin cancer (basal cell carcinoma or squamous cell carcinoma), then research staff will contact participants by phone and ask them to visit their general practitioners for immediate clinical assessment. Participants are informed they will be provided with their telediagnosis results at the CSE if there is no urgent action required prior to that appointment.

\section{Control Group (Naked-Eye SSE)}

Participants in the naked-eye SSE group are also asked to conduct SSEs at baseline, 1 and 2 months. They do not use mobile teledermoscopy during their SSE, but instead receive e-mailed web-based instructions on what to look for using the AC rule and how to complete a whole-body SSE. SSEs in this group will be aided by another person (i.e., partner, a relative or close friend) to allow for checking hard-to- 


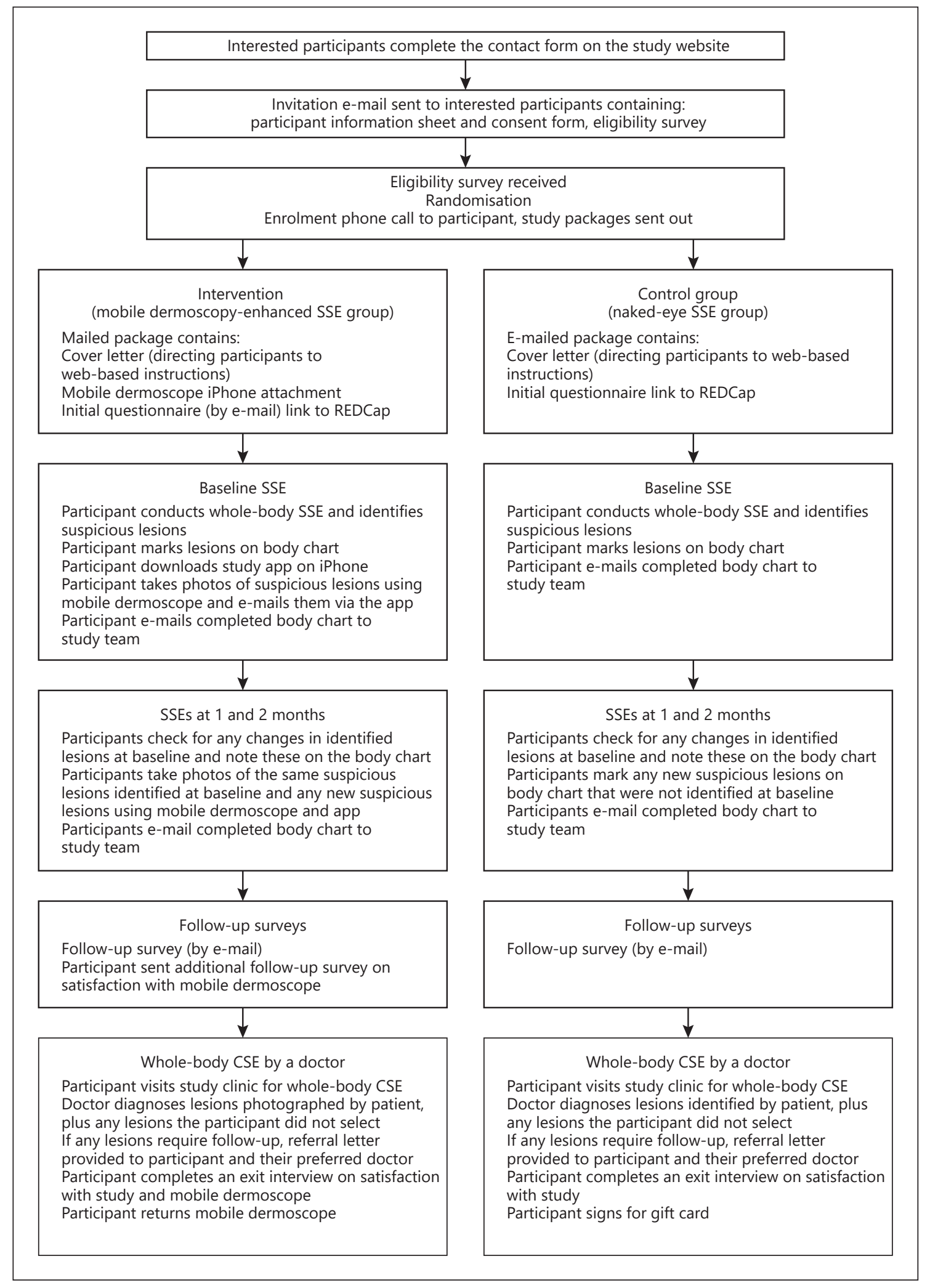

Fig. 1. Overview of study procedures. 
see areas of the body. On the body chart participants will note the location of each lesion, and number skin lesions they think need management by a doctor as well as their concern (index of suspicion) for the urgency for management (from 1 not at all concerned to 10 very concerned). Participants in the naked-eye SSE group will e-mail their completed body chart to the research team after every skin check to allow assessment for adherence. On the body chart, participants can include other notes, such as history of each lesion, whether it changed or symptoms (i.e., itch, pain, bleeding).

\section{Questionnaires}

Initial Questionnaire

All questionnaires will be created and distributed using REDCap software. Participants will complete an online initial questionnaire assessing:

- Demographics (7 questions): age, education, work, work type (indoors or outdoors), marital status, private health insurance and postcode; questions were adapted from our previous studies [18]

- Previous CSE history (8 questions): history of a CSE and year it was conducted; who initiated the CSE; doctor's recommendation to conduct SSE or instruction for how to do an SSE; lesions requiring management, lesions biopsied or excised by the doctor (if so how many) and recommendation of follow-up CSE [23, 24]

- Confidence conducting SSE and taking photos of skin lesions (1 question): confidence with checking their skin for skin cancer ranging from 1 (not at all confident) to 10 (highly confident) adapted from our previous study [18]

- Previous SSE history (5 questions): examination of their own skin in the past 12 months (yes, no, don't know), extent of the SSE (skin spots only, part of body, whole body); times SSE conducted within last 3 months $(0,1-2,3-4,5-6$ times, more than 6 times); plan to conduct SSE in the next 12 months (yes, no, don't know), current concern about a spot or mole (yes, no, unsure) [24]

- Use of smartphones (5 questions): use of a smartphone before, i.e. overall skill level (very unskilled, unskilled, skilled, very skilled), frequency of smartphone use for phone calls and Internet browsing (several times a day, once a day, several times a week, hardly ever) and use for online activities [25]

- Attitudes towards SSE (12-item Likert scale): thoughts on checking their skin ranging from strongly agree to strongly disagree $[7,23]$; the scale has been validated using item-response theory [26]

- Thoughts about melanoma scale (12-item Likert scale): this is derived from existing scales that measure cancer worry [27, 28]; it reflects on frequency and extent of worry, effect of worry on mood and daily activities, and symptom-based worry on a 5 -point Likert scale (never, rarely, sometimes, often, always)

- Quality of life (EQ-5D-5L) [29]. The descriptive system comprises 5 dimensions: mobility, self-care, usual activities, pain/ discomfort and anxiety/depression; each dimension has 5 levels: no problems, slight problems, moderate problems, severe problems and extreme problems

- Perception of melanoma risk (3 questions): likelihood of developing melanoma in the next 10 years, likelihood of developing melanoma compared to others and likelihood of being diagnosed with a skin cancer at some time in the future; responses will be recorded on a 5-point Likert scale

- Mobile teledermoscopy acceptance (27 items): this will be measured using a previously validated survey [30] which assesses the following technology acceptance model domains: perceived usefulness (5 items), perceived ease of use (4 items), attitude and intention (6 items), compatibility (4 items), facilitators (3 items), subjective norms (3 items) and trust (2 items). Respondents will be asked to rate their agreement on a 5 -point Likert scale (from $5=$ strongly agree to $1=$ strongly disagree)

\section{Follow-Up Questionnaire}

Participants in both groups will be asked to complete an online follow-up questionnaire which addresses:

- Willingness to pay for mobile teledermoscopy-enhanced SSE compared to standard care [31]. The discrete choice experiment question set was comprised of 24 questions, divided into 2 blocks ( 12 questions per participant); this survey asks respondents to make 12 choices between skin cancer screening options (skin cancer clinics, mobile teledermoscopy-enhanced SSE, naked-eye SSE) taking into account waiting times, costs and diagnosing clinician

- Mobile teledermoscopy acceptance, thoughts about melanoma scale, confidence conducting SSE and quality of life (EQ-5D-5L) (repeated from initial questionnaire)

Participants in the intervention group will be asked additional questions developed in our previous pilot study [18] including: satisfaction with study instructions, ease of use and satisfaction with the dermoscope, any technological difficulties encountered and intentions for future use of the technology. An open-ended question at the end of the questionnaire will request any further feedback about the study from both groups.

\section{Clinical Skin Examination}

Participants will be asked to attend the clinic at the QUT, Kelvin Grove Campus, for a whole-body CSE by a doctor. The doctor will first assess the consumer-selected lesions, then will conduct a whole-body CSE to assess whether the participant missed any lesions that would require monitoring or treatment. The CSE will be conducted by the dermatologist (H.P.S.). In the case of lesions that require biopsy or further management, the study team will write a referral letter to the participant's general practitioner. The study team will enter data on the clinical diagnoses of any lesions identified during the CSE and their management (none, observation, treatment [surgical or non-surgical], referral). Where applicable, a copy of the histopathological report will be requested from the general practitioner with the consent of the participant. Following completion of the CSE, an in-person individual interview with participants will be conducted on their study experience and opinions on mobile teledermoscopy-enhanced SSE. The intervention group participants return the dermoscope at the appointment.

\section{Participant Reimbursement}

After the CSE and interview, participants will be provided with a 50-AUD gift card to reimburse them for their time, data costs for app usage and travel expenses.

\section{Outcomes and Analysis}

Primary Analysis

Primary outcome measures include (Table 1):

(a) body sites examined, lesions photographed and missed;

(b) sensitivity of mobile teledermoscopy-enhanced SSE versus in-person CSE using either participants or lesions as denominator; 
Table 1. Overview of health outcomes measured and analysis

\begin{tabular}{|c|c|}
\hline Outcomes & Measures and methods \\
\hline \multicolumn{2}{|l|}{ Primary outcomes } \\
\hline Sensitivity & $\begin{array}{l}\text { Sensitivity of mobile teledermoscopy-enhanced SSE versus naked-eye SSE. The reference standard is a } \\
\text { clinical skin examination conducted by a dermatologist } \\
\text { True positive/(true positive + false negative) }\end{array}$ \\
\hline Specificity & $\begin{array}{l}\text { Specificity of mobile teledermoscopy-enhanced SSE versus naked-eye SSE. The reference standard is a } \\
\text { CSE conducted by a dermatologist } \\
\text { True negative/(true negative + false positive) }\end{array}$ \\
\hline Overall concordance & Participant identified lesions versus CSE by dermatologist \\
\hline \multicolumn{2}{|l|}{ Secondary analysis } \\
\hline Cost-effectiveness analysis & Willingness to pay for teledermoscopy diagnoses compared to standard care \\
\hline Quality of life & $\begin{array}{l}\text { The EQ-5D-5L descriptive system is scored using established methods and converted into a single } \\
\text { index value, facilitating the calculation of quality-adjusted life years [29] }\end{array}$ \\
\hline Consumer satisfaction & Frequencies \\
\hline Thoughts about melanoma & Median, frequencies, $\chi^{2}$ or Fisher's exact test \\
\hline
\end{tabular}

SSE, skin self-examination; CSE, clinical skin examination.

(c) concordance of telediagnosis with clinical diagnosis, as well as histological diagnosis for any lesions excised or biopsied.

Analyses will be conducted according to intention-to-treat analysis, keeping all data from the participants within their assigned SSE instruction groups. We will summarise participants' characteristics, number and type of lesions sent via mobile teledermoscopy-enhanced SSE, found at CSE or missed (considered worthwhile photographing or further clinical monitoring at CSE), using descriptive statistics, and compare the proportions of lesions submitted for telediagnosis and missed within the mobile teledermoscopy-enhanced SSE and naked-eye SSE groups using the $\chi^{2}$ test. We will calculate sensitivity and specificity by utilising $2 \times 2$ tables cross-referencing the tele- and CSE diagnoses. A skin lesion will be counted as positive if it required further intervention including close monitoring, biopsy, excision or other treatment, and negative if it did not require such interventions.

We will calculate sensitivity and specificity both at participant and at lesion level adjusting the latter for clustering of lesions within participants as described by Genders et al. [32]. Appropriate methods will be used for estimating sensitivities and specificities for the longitudinal data. The longitudinal 2nd and 3rd SSEs add another level of clustering (time), and we will use mixed/generalised estimating equation models to assess improvements in sensitivity over time.

We will also assess the concordance of the telediagnosis and the dermatologists' in-person clinical diagnoses, and any lesions excised or biopsied will be compared to histology using kappa statistics.

Briefly, we will conduct the analysis on two different levels:

1 Participant-level analysis: all participants will be instructed to look at their body and note any lesions they are concerned about; if they find one such concerning lesion and if this or any other lesion is also found suspicious on clinical examination, they will receive a correct positive score, as this analysis assumes that a telediagnosis of a suspicious lesion would result in a clinical whole-body examination and any other lesions would be found; this method assumes the doctor will detect other lesions during the follow-up consultation if indeed such lesions are present

2 Lesion-level analysis: in this analysis only those lesions that the participant identified as suspicious confirmed by the doctor will be counted as correct positive, any that the participant overlooks and are then found at the clinical visit will contribute to the false-negative count

Kappa statistics will determine agreement (corrected for chance) between tele- and CSE diagnoses with and without accounting for differential diagnoses.

\section{Secondary Analysis}

We will undertake a cost-effectiveness analysis alongside the randomised controlled trial to assess the value for money provided by mobile teledermoscopy-enhanced SSE compared to naked-eye SSE. We will then perform a modelled cost-effectiveness analysis to extrapolate the randomised trial data to compare the long-term value for money of implementing mobile teledermoscopy-enhanced SSE within mobile teledermoscopy models for screening, triage or diagnosis.

Willingness to Pay. This discrete choice experiment was designed using the principle of $d$-efficiency and will be analysed using a mixed logit model. A mixed logit model was used to estimate preferences for skin cancer screening services, marginal willingness to pay for a teledermoscopy service and probability of service uptake.

Satisfaction with Services. Descriptive analysis will be used to assess satisfaction with the mobile dermoscope as self-reported by participants in the follow-up questionnaire.

\section{Sample Size}

To account for ineligible participants, refusal rates and attrition, an estimated 480 potential participants will be assessed for
16

Dermatology 2019;235:11-18

DOI: $10.1159 / 000493729$
Janda/Horsham/Koh/Gillespie/Loescher/ Vagenas/Soyer 
eligibility, and 230 will be enrolled. Recruitment of this number is achievable over an 18-month recruitment period as in our previous study 55 patients were enrolled over a 3-month period from a once-off traditional media call only. Expecting a participant dropout of $5 \%$ based on our previous studies $[18,26]$, a sample size of 110 at analyses in each group will provide more than $80 \%$ power to detect an improvement in sensitivity by $20 \%$ or greater [33]. Such improvement is clinically relevant and within the maximum achieved in previous studies that investigated SSE aids $[9,10]$.

\section{Data Collection and Management}

All data collected from participants will remain confidential at all times. Each participant will be assigned a study number, unique, but otherwise meaningless. This number together with the participants' identifying information (name, address and telephone contact) will be stored separately from all other study materials on a password-protected server, only accessible by senior research staff. On all other documents we will use the participant's study number only, and thus all these materials are only potentially reidentifiable. The information collected needs to be in potentially re-identifiable form so that data collected via multiple methods (i.e., images of lesions e-mailed by participants, and data collected by questionnaire) can be linked. Data will be recorded on the REDCap system, a secure study database.

Expected Findings, Implications and Dissemination

We expect the trial will prove to be both feasible and acceptable to participants. We will submit the findings of our research for publication in peer-reviewed journals, and the findings will also be presented at national and international conferences. If we find that mobile teledermoscopy-enhanced SSE leads to an increase in sensitivity, this may inform future public health guidelines and communication information materials.

\section{Trial Progress}

The study is registered on the Australian and New Zealand Trial Registry (ANZCTR) on July 27, 2016, the first participant was recruited on March 6, 2017, enrolment is expected to be completed in August 2018, with data analysis completed by December 2018.

\section{Conclusions}

Mobile teledermoscopy is commercially available and could lead to effective triage of high-risk lesions and potentially be life-saving; however, work is required to allow patients and clinicians to take full advantage of the technology. Despite promising features, mobile teledermoscopy has not yet been translated into service delivery to the extent that may be expected, possibly due to the lack of proof for cost-effectiveness or store-and-forward dermatology business models. This study seeks to overcome these technology, clinician and systems barriers and will therefore be an important step towards redesigning skin cancer early detection and care in Australia.

\section{Key Message}

This is the first trial to directly compare technology-enhanced and naked-eye skin self-examination.

\section{Acknowledgments}

We would like to thank the following research staff: Naomi Stekelenburg, Linda Finch and Centaine Snoswell. This project is being undertaken as part of a research project on behalf of QUT and the University of Queensland, in partnership with FotoFinder PTY, the Princess Alexandra Hospital Foundation, Melanoma Patients Australia, the Skin and Cancer Foundations (VIC, NSW, QLD), and the Dermatology Departments of the University of Graz, University of Arizona and Memorial Sloan Kettering Cancer Center, USA.

\section{Statement of Ethics}

This study has been approved by the QUT's Human Research Ethics Committee on June 20, 2016 (QUT approval No.: 1400000807).

\section{Disclosure Statement}

H. Peter Soyer is a shareholder of e-derm consult $\mathrm{GmbH}$ and MoleMap by Dermatologists Ltd Pty. He provides teledermatological reports regularly for both companies. He is also advisor of First Derm ${ }^{\mathrm{TM}}$.

\section{Funding Sources}

The study is funded by a research grant awarded to M.J. from the National Health and Medical Research Council APP1113962. M.J. is funded by a TRIP Fellowship APP1151021.

\section{Author Contributions}

All authors made contributions to the development of the trial protocol and have been involved in drafting this manuscript or revising it critically for important intellectual content. M.J. is the overall chief investigator. H.P.S. provides dermatological expertise including providing teledermoscopy reports of all study lesions and performing all CSEs. M.J. and D.V. provide statistical expertise. N.G. leads on examining pathways to integrate mobile teledermoscopy into practice and business models for teledermoscopy. L.J.L. provides protocol review. C.H. and U.K. are the project coordinators. All authors have given final approval of this paper. 


\section{References}

1 Australian Institute of Health and Welfare. Cancer in Australia: An overview 2014. Cancer series No 90. Cat. No CAN 88. Canberra: AIHW; 2014.

2 Green AC, Baade P, Coory M, Aitken JF, Smithers M. Population-based 20-year survival among people diagnosed with thin melanomas in Queensland, Australia. J Clin Oncol. 2012 May;30(13):1462-7.

3 Rigel DS, Carucci JA. Malignant melanoma: Prevention, early detection, and treatment in the 21st century. CA Cancer J Clin. 2000 JulAug;50(4):215-36.

4 American Cancer Society. Skin cancer prevention and early detection.

5 Cancer Council Australia. Check for signs of skin cancer. 2018

6 Youl PH, Baade PD, Janda M, Del Mar CB, Whiteman DC, Aitken JF. Diagnosing skin cancer in primary care: how do mainstream general practitioners compare with primary care skin cancer clinic doctors? Med J Aust. 2007 Aug;187(4):215-20.

7 Aitken JF, Janda M, Lowe JB, Elwood M, Ring IT, Youl PH, et al. Prevalence of whole-body skin self-examination in a population at high risk for skin cancer (Australia). Cancer Causes Control. 2004 Jun;15(5):453-63.

8 Olsen CM, Thompson BS, Green AC, Neale RE, Whiteman DC; QSkin Sun and Health Study Group. Sun Protection and Skin Examination Practices in a Setting of High Ambient Solar Radiation: A Population-Based Cohort Study. JAMA Dermatol. 2015 Sep;151(9): 982-90.

9 Hamidi R, Peng D, Cockburn M. Efficacy of skin self-examination for the early detection of melanoma. Int J Dermatol. 2010 Feb;49(2): 126-34.

10 King AJ, Gehl RW, Grossman D, Jensen JD. Skin self-examinations and visual identification of atypical nevi: comparing individual and crowdsourcing approaches. Cancer Epidemiol. 2013 Dec;37(6):979-84.

11 Muhn CY, From L, Glied M. Detection of artificial changes in mole size by skin self-examination. J Am Acad Dermatol. 2000 May;42(5 Pt 1):754-9.
12 Luttrell MJ, McClenahan P, HofmannWellenhof R, Fink-Puches R, Soyer HP. Laypersons' sensitivity for melanoma identification is higher with dermoscopy images than clinical photographs. Br J Dermatol. 2012 Nov;167(5):1037-41.

13 Loescher LJ, Janda M, Soyer HP, Shea K, Curiel-Lewandrowski C. Advances in skin cancer early detection and diagnosis. Semin Oncol Nurs. 2013 Aug;29(3):170-81.

14 Marghoob AA, Scope A. The complexity of diagnosing melanoma. J Invest Dermatol. 2009 Jan;129(1):11-3.

15 Vestergaard ME, Macaskill P, Holt PE, Menzies SW. Dermoscopy compared with naked eye examination for the diagnosis of primary melanoma: a meta-analysis of studies performed in a clinical setting. Br J Dermatol. 2008 Sep;159(3):669-76.

16 Janda M, Soyer HP. Automated diagnosis of melanoma. Med J Aust. 2017 Oct;207(8):3612

17 Janda M, Loescher LJ, Banan P, Horsham C, Soyer HP. Lesion selection by melanoma high-risk consumers during skin self-examination using mobile teledermoscopy. JAMA Dermatol. 2014 Jun;150(6):656-8.

18 Manahan MN, Soyer HP, Loescher LJ, Horsham C, Vagenas D, Whiteman DC, et al. A pilot trial of mobile, patient-performed teledermoscopy. Br J Dermatol. 2015 Apr;172(4): 1072-80.

19 Lim D, Oakley AM, Rademaker M. Better, sooner, more convenient: a successful teledermoscopy service. Australas J Dermatol. 2012 Feb;53(1):22-5

20 Marchetti MA, Fonseca M, Dusza SW, Scope A, Geller AC, Bishop M, et al. Dermatoscopic imaging of skin lesions by high school students: a cross-sectional pilot study. Dermatol Pract Concept. 2015 Jan;5(1):11-28.

21 Wu X, Oliveria SA, Yagerman S, Chen L, DeFazio J, Braun R, et al. Feasibility and Efficacy of Patient-Initiated Mobile Teledermoscopy for Short-term Monitoring of Clinically Atypical Nevi. JAMA Dermatol. 2015 May;151(5): 489-96.

22 Luttrell MJ, Hofmann-Wellenhof R, Fink-Puches R, Soyer HP. The AC Rule for melanoma: a simpler tool for the wider community. J Am Acad Dermatol. 2011 Dec;65(6):1233-4.
23 Janda M, Baade PD, Youl PH, Aitken JF, Whiteman DC, Gordon L, et al. The skin awareness study: promoting thorough skin self-examination for skin cancer among men 50 years or older. Contemp Clin Trials. 2010 Jan;31(1):119-30.

24 Aitken JF, Youl PH, Janda M, Elwood M, Ring IT, Lowe JB. Comparability of skin screening histories obtained by telephone interviews and mailed questionnaires: a randomized crossover study. Am J Epidemiol. 2004 Sep; 160(6):598-604.

25 Pew Research Center's Internet \& American Life Project. Available from: http://www. pewinternet.org.

26 Djaja N, Youl P, Aitken J, Janda M. Evaluation of a skin self examination attitude scale using an item response theory model approach. Health Qual Life Outcomes. 2014 Dec;12(1): 189

27 Loescher LJ. Cancer worry in women with hereditary risk factors for breast cancer. Oncol Nurs Forum. 2003 Sep-Oct;30(5):767-72.

28 Lerman C, Daly M, Masny A, Balshem A. Attitudes about genetic testing for breast-ovarian cancer susceptibility. J Clin Oncol. 1994 Apr;12(4):843-50.

29 Herdman M, Gudex C, Lloyd A, Janssen M, Kind P, Parkin D, et al. Development and preliminary testing of the new five-level version of EQ-5D (EQ-5D-5L). Qual Life Res. 2011 Dec;20(10):1727-36.

30 Horsham C, Loescher LJ, Whiteman DC, Soyer HP, Janda M. Consumer acceptance of patient-performed mobile teledermoscopy for the early detection of melanoma. Br J Dermatol. 2016 Dec;175(6):1301-10.

31 Spinks J, Janda M, Soyer HP, Whitty JA. Consumer preferences for teledermoscopy screening to detect melanoma early. J Telemed Telecare. 2016 Jan;22(1):39-46.

32 Genders TS, Spronk S, Stijnen T, Steyerberg EW, Lesaffre E, Hunink MG. Methods for calculating sensitivity and specificity of clustered data: a tutorial. Radiology. 2012 Dec;265(3): 910-6.

33 Li J, Fine J. On sample size for sensitivity and specificity in prospective diagnostic accuracy studies. Stat Med. 2004 Aug;23(16):2537-50. 